\title{
Editor's note on the themed issue: integration of machine learning and quantitative systems pharmacology
}

\author{
Peter L. Bonate ${ }^{1}$
}

Published online: 18 January 2022

(C) The Author(s), under exclusive licence to Springer Science+Business Media, LLC, part of Springer Nature 2022

In a few short years, quantitative systems pharmacology (QSP) has become a major tool available to pharmacometricians to improve decision making in drug development, so much so that today pharmacometrics can be broadly classified into three groups: population-based methods, physiological-based pharmacokinetics (PBPK), and QSP. Recently, we are starting to see the emergence of a fourth field: machine learning (ML). These recent ML papers have primarily used ML with population-based methods. There are papers on the use of genetic algorithms to improve population-based model selection [1-3], on using ML to identify important covariates in covariate selection [4], the use of recurrent neural networks to predict pharmacodynamic profiles [5], and the use of ML to help guide precision dosing in patients [6].

But machine learning is nothing new. In fact, it's QSP that is new. QSP had its official introduction with the rollout of a white paper from the National Institutes of Health in 2011 [7], just a little over a decade ago. But ML is much older, having its origins in the 1950s when the first ML algorithms were developed by IBM for the game of checkers [8]. The basic unit of neural networks, the perceptron, which is the foundation for deep learning, was developed in 1957. Backpropagation was developed in the 1960s. Going back to the 1980s and 1990s, one can find the first ML papers applied to PKPD modeling [9-11]. But the use of ML never gained any traction over the populationmethods that were also being developed at the same time and it remained a niche area in pharmacometrics for decades. It's safe to say that overall, neural networks and ML didn't take off until the 21 st century when computing power and huge amounts of data converged to finally take

Peter L. Bonate

Peter.bonate@astellas.com

1 Astellas, Northbrook, USA full advantage of these algorithms, which require huge amounts of data and fast processing speed to be useful.

As part of the on-going efforts of the Journal of Pharmacokinetics and Pharmacodynamics (JPKPD) to promote the application of ML in pharmacometrics, this issue presents a series of papers related to the application of ML to augment or assist in the development and analysis of QSP models. The lead paper in this issue is an industry-academia white paper from the International Society of Pharmacometrics Working Group on the Integration of Quantitative Systems Pharmacology and Machine Learning [12]. The working group has identified four areas where machine learning can be used with QSP: parameter estimation or extraction, model structure, dimension reduction, and virtual populations. Each area is reviewed with some specific case studies highlighted. The paper concludes with some general thoughts on the future of this integration, some challenges, and some of the practical considerations in implementing ML in industry.

Following the white paper are a series of original research articles and reviews that highlight the application of ML to specific functional areas:

- Aghamiri, Amin, and Helikar [13] present a general review of the application of ML to QSP.

- Cheng et al. [14] present a focused review on the use of ML and deep learning (which is a subset of machine learning, much like machine learning is a subset of artificial intelligence, that uses neural networks for learning) to diagnose and model heart failure.

- Generational adversarial networks (GANs) are a type of deep learning method where two neural networks compete against one another in a type of zero-sum game. GANs have been in the news recently for their ability to generate extremely realistic images of people that do not exist. Parikh et al. [15] use GANs to generate virtual populations of cardiac myocyte models and applied it used it to study the positive ionotropic effects of omecamtiv mecarbil. 
- Bayesian Networks (BN) are probabilistic models that explain how a set of covariates interact with each other. Using metabolic and cardiovascular markers extracted from the National Heath and Nutritional Examination Survey (NHANES) database, McComb et al. [16] use Bayesian networks to first understand the relationship between these markers and then used ML to to model disease progression as a person ages, essentially using current and previous measurements to predict future measurements. Such a model could be used by clinicians in the future to monitor long term treatment responses to diabetes or cardiovascular drugs.

- Network analysis is a methodology for examining the relationships among high dimensional biological data. In a sense, its output is similar to BN, but operates on a much larger scale. Hayes, Sachs, and Cho [17] present a new network analysis method, called DEKER-NET, which uses ML to identify relationships among covariates. Using Dialogue for Reverse Engineering Assessments and Methods (DREAM) challenge data, the authors show that DEKER-NET performs as good or better than existing methods.

- Mager and colleagues first introduced Boolean network models to pharmacometrics just a few years ago by demonstrating how this new methodology can be used to build and simplify QSP models prior to development of more formal ordinary differential equation-based models [18-20]. Putnins et al. [21] further develop this methodology by implementing a pipeline approach to simplify and standardize network model development. They demonstrate their approach by developing a QSP model for flotetuzumab.

- Zhang and Tyson [22] present a novel approach, using ML followed by bifurcation analysis, to generate virtual patients in QSP models. ML can quickly examine thousands of virtual patients and determine which parameters are important explaining variability in the data; bifurcation analysis can provide greater mechanistic insight into those parameters identified as important. The authors illustrate their method using a QSP model of the hypothalamic-pituitary-adrenal axis.

Science progresses. It evolves. New knowledge is created. Pharmacometrics is no exception. This issue demonstrates that as both QSP and ML evolve and mature, we are starting to see the merging or cross-pollination of two disciplines to create new opportunities for research and further growth of both fields. The editors of the Journal and organizers of this issue (Carolyn Cho, Tongli Zhang, and myself) would like to thank all the authors and reviewers for their help in making this issue a success.

\section{References}

1. Ismail M, Sale M, Yu Y, Liu S, Pflug B, Bies RR (2021) Development of a genetic algorithm and NONMEM workbench for automating and improving population pharmacokinetic/pharmacodynamic model selection. J Pharmacokinet Pharmacodyna (online ahead of print)

2. Sherer EA, Sale ME, Pollock BG, Belani CP, Egorin MJ, Ivy PS, Lieberman JA, Manuck SB, Marder SR, Muldoon MF, Scher HI, Solit DB, Bies RR (2012) Application of a single-objective, hybrid genetic algorithm approach to pharmacokinetic model building. J Pharmacokinet Pharmacodyn 39:393-414

3. Bies RR, Muldoon MF, Pollock BG, Manuck S, Smith G, Sale ME (2006) A genetic algorithm-based, hybrid machine learning approach to model selection. J Pharmacokinet Pharmacodyn 33:195-221

4. Sibieude E, Khandelwal A, Hesthaven JS, Girard P, Terranova N (2021) Fast screening of covariates in population models empowered by machine learning. J Pharmacokinet Pharmacodyn 48:597-609

5. Liu X, Liu C, Huang R, Zhu H, Liu Q, Mitra S, Wang Y (2021) Long short-term memory recurrent neural network for pharmacokinetic-pharmacodynamic modeling. Int J Clin Pharmacol Ther 59:138-146

6. Woillard J-B, Labriffe M, Debord J, Marquet P (2020) Tacrolimus exposure prediction using machine learning. Clin Pharmacol Ther 110:361-369

7. Sorger PK Chair, Allerheiligen SRB co-chair, Abernethy DR, Altman RB, Brouwer KLR, Califano A, D'Argenio DZ, Iyengar R, Jusko WJ, Lalonde R, Lauffenburger DA, Shoichet B, Stevens JL, Subramaniam S, van der Graaf PH, Vicini P (2011) Quantitative and systems pharmacology in the post-genomic era: new approaches to discovering drugs and understanding therapeutic mechanisms: an NIH white paper by the QSP workshop group. (https://www.nigms.nih.gov/training/documents/system spharmawpsorger2011.pdf. Accessed 7 Jan 2022

8. Foote KD (2021) A brief history of machine learning. (https:// www.dataversity.net/a-brief-history-of-machine-learning/\#. Accessed 7 Jan 2022

9. Gobburu JVS, Chen EP (1996) Artificial neural networks as a novel approach to integrated pharmacokinetic-pharmacodynamic analysis. J Pharm Sci 85:505-510

10. Erb RJ (1995) The backpropagation neural network-a Bayesian classifier. Introduction and applicability to pharmacokinetics. Clin Pharmacokinet 29:69-79

11. Veng-Pedersen P, Modi NB (1993) Application of neural networks to pharmacodynamics. J Pharm Sci 82:918-926

12. Zhang T, Androulakis IP, Bonate PL, Cheng L, Helikar T, Parikh J, Rackauckas C, Subramanian K, Cho CR (2021) Two heads are better than one: current landscape of integrating QSP and machine learning. J Pharmacokinet Pharmacodyn 49

13. Aghamiri SS, Amin R, Helikar T (2022) Recent applications of quantitative systems pharmacology and machine learning models across diseases. J Pharmacokinet Pharmacodyn. https://doi.org/ 10.1007/s10928-021-09790-9

14. Cheng L, Qiu Y, Schmidt B, Wei G-W (2022) Review of applications and challenges of quantitative systems pharmacology modeling and machine learning for heart failure. J Pharmacokinet Pharmacodyn. https://doi.org/10.1007/s10928-02109785-6

15. Parikh J, Rumbell T, Butova X, Myachina T, Acero JC, Khazim S, Solovyova O, Kozloski J, Khokhlova A, Gurev V (2022) Generative adversarial networks for construction of virtual populations of mechanistic models: simulations to study omecamtiv mecarbil action. J Pharmacokinet Pharmacodyn 49 
16. McComb M, Hageman-Blair R, Lysy M, Ramanathan M (2022) Machine learning-guided, big data-enabled, biomarker-based systems pharmacology: modeling the stochasticity of natural history and disease progression. J Pharmacokinet Pharmacodyn. https://doi.org/10.1007/s10928-021-09786-5

17. Hayes SMS, Sachs JR, Cho CR (2022) From complex data to biological insight: 'DEKER' feature selection and network inference. J Pharmacokinet Pharmacodyn. https://doi.org/10. 1007/s10928-021-09792-7

18. Chudasama VL, Ovacik MA, Abernethy DR, Mager DE (2015) Logic-based and cellular pharmacodynamic modeling of bortezomib responses in U266 human myeloma cells. J Pharmacol Exp Ther 354:448-458

19. Ramakrishnan V, Mager DE (2018) Network-based analysis of bortezomib pharmacodynamic heterogeneity in multiple myeloma cells. J Pharmacol Exp Ther 365:734-751
20. Bloomingdale P, Nguyen VA, Niu J, Mager DE (2018) Boolean network modeling in systems pharmacology. J Pharmacokinet Pharmacodyn 45:159-180

21. Putnins M, Campagne O, Mager DE, Androulakis IP (2022). From data to QSP models: A pipeline for using Boolean networks for hypothesis inference and dynamic model building. J Pharmacokinet Pharmacodyn. https://doi.org/10.1007/s10928-02109797-2

22. Zhang T, Tyson JJ (2022) Understanding virtual patients efficiently and rigorously by combining machine learning with dynamical modelling. J Pharmacokinet Pharmacodyn. https://doi. org/10.1007/s10928-021-09798-1

Publisher's Note Springer Nature remains neutral with regard to jurisdictional claims in published maps and institutional affiliations. 